\title{
Reliability and validity of gait analysis by android-based smartphone.
}

\section{$\operatorname{AUTHOR}(\mathrm{S}):$}

Nishiguchi, Shu; Yamada, Minoru; Nagai, Koutatsu; Mori, Shuhei; Kajiwara, Yuu; Sonoda, Takuya; Yoshimura, Kazuya; ... Muto, Shinyo; Ishihara, Tatsuya; Aoyama, Tomoki

\section{CITATION:}

Nishiguchi, Shu ...[et al]. Reliability and validity of gait analysis by android-based smartphone.. Telemedicine and e-health 2012, 18(4): 292-296

\section{ISSUE DATE:}

2012-05

\section{URL:}

http://hdl.handle.net/2433/156135

\section{RIGHT:}

This is a copy of an article published in the Telemedicine and e-health.; (c) 2012 Mary Ann Liebert, Inc.; "Telemedicine and e-Health" is available online at: http://www.liebertonline.com. 


\section{Reliability and Validity of Gait Analysis by Android-Based Smartphone}

Shu Nishiguchi, R.P.T., ${ }^{1}$ Minoru Yamada, R.P.T., Ph.D., ${ }^{1}$ Koutatsu Nagai, R.P.T., M.S., ${ }^{1}$ Shuhei Mori, R.P.T., Yuu Kajiwara, R.P.T., ${ }^{1}$ Takuya Sonoda, R.P.T., ${ }^{1}$ Kazuya Yoshimura, R.P.T., Hiroyuki Yoshitomi, M.D., Ph.D., ${ }^{2}$ Hiromu Ito, M.D., Ph.D., ${ }^{3}$ Kazuya Okamoto, Ph.D., ${ }^{4}$ Tatsuaki Ito, M.E., ${ }^{5}$ Shinyo Muto, M.E., Tatsuya Ishihara, M.E., and Tomoki Aoyama, M.D., Ph.D. ${ }^{1}$

${ }^{1}$ Department of Physical Therapy, Human Health Sciences, ${ }^{2}$ Department of Orthopaedic Surgery, and ${ }^{3}$ Department for the Control of Rheumatic Diseases, Kyoto University Graduate School of Medicine, Kyoto, Japan.

${ }^{4}$ Department of Medical Informatics, Kyoto University Hospital, Kyoto, Japan.

${ }^{5} \mathrm{NTT}$ Cyber Solutions Laboratories, Kanagawa, Japan.

\section{Abstract}

Smartphones are very common devices in daily life that have a builtin tri-axial accelerometer. Similar to previously developed accelerometers, smartphones can be used to assess gait patterns. However, few gait analyses have been performed using smartphones, and their reliability and validity have not been evaluated yet. The purpose of this study was to evaluate the reliability and validity of a smartphone accelerometer. Thirty healthy young adults participated in this study. They walked $20 \mathrm{~m}$ at their preferred speeds, and their trunk accelerations were measured using a smartphone and a tri-axial accelerometer that was secured over the L3 spinous process. We developed a gait analysis application and installed it in the smartphone to measure the acceleration. After signal processing, we calculated the gait parameters of each measurement terminal: peak frequency (PF), root mean square (RMS), autocorrelation peak (AC), and coefficient of variance (CV) of the acceleration peak intervals. Remarkable consistency was observed in the test-retest reliability of all the gait parameter results obtained by the smartphone $(\mathrm{p}<0.001)$. All the gait parameter results obtained by the smartphone showed statistically significant and considerable correlations with the same parameter results obtained by the tri-axial accelerometer $(P F \mathrm{r}=0.99$, RMS $\mathrm{r}=0.89$, AC $\mathrm{r}=0.85, \mathrm{CV} \mathrm{r}=0.82 ; \mathrm{p}<0.01$ ). Our study indicates that the smartphone with gait analysis application used in this study has the capacity to quantify gait parameters with a degree of accuracy that is comparable to that of the tri-axial accelerometer.

Key words: smartphones, accelerometers, gait analysis, reliability, validity

\section{Introduction}

W

alking is a natural form of movement, and most people walk every day to perform a variety of common tasks such as shopping and traveling. This most natural capability, however, is commonly influenced by major cerebral impairments, ${ }^{1}$ severe musculoskeletal disorders, ${ }^{2}$ and aging. ${ }^{3}$

The study of human movement, an area that has been actively researched for many years, has sought to identify and characterize gait patterns. For years, the quantitative analysis of gait patterns has been studied in gait laboratories that are equipped with many sophisticated measurement and analysis devices such as ground reaction force plates and three-dimensional kinematic motion analysis systems. ${ }^{4,5}$ However, the use of such facilities requires specialized personnel and laboratory environment. Moreover, most of the equipment is costly, and the data acquisition procedures are often cumbersome. In fact, for some time now, the use of facilities for gait analysis has been limited to field research and clinical settings.

More recently, wireless tri-axial accelerometers are being used widely for gait analysis because they are easy to use and inexpensive and they do not require a laboratory environment. Henriksen et al. ${ }^{6}$ found the reliability of trunk accelerometric gait analysis to be satisfactory, as it yielded high values for intraclass correlation coefficient (ICC) and low values for measurement error and coefficients of variation. By conducting a personal computer analysis of the raw acceleration data collected from a person's gait, it is possible to quantify the variability, regularity, and rhythmic pattern of the person's gait. ${ }^{7}$ Accelerometric gait analysis enables us to assess gait patterns from the perspective of gait stability, which is different from the previously used kinematic approach.

A current and quite recent trend has seen the deployment of accelerometers in off-the-shelf cellphone handsets such as smartphones, and studies of gait analysis and measurements of anticipatory postural adjustments have been attempted using a smartphone accelerometer. ${ }^{8,9}$ Lemoyne et al. ${ }^{9}$ performed gait analysis experiments using a smartphone that demonstrated the capacity to accurately quantify gait parameters with a sufficient level of consistency. Thus, smartphones may have the potential to assess gait patterns as competently as accelerometers. However, gait analysis using smartphones has not been explored widely, and its reliability and validity have not yet been evaluated. Therefore, the purpose of this study was to evaluate the reliability and validity of a smartphone accelerometer. In this study, gait parameters obtained by a 


\section{GAIT ANALYSIS BY ANDROID-BASED SMARTPHONE}

smartphone were compared with those obtained by a conventional accelerometer.

\section{Subjects and Methods SUBJECTS}

Students at Kyoto University were recruited as subjects for this study. 17 men and 13 women volunteered, none of whom reported present or previous diseases or injuries associated with gait and/or balance impairments. Informed consent was obtained from all subjects prior to their participation, in accordance with the guidelines approved by the Kyoto University Graduate School of Medicine (approval number E1095) and the Declaration of Human Rights, Helsinki, 1975.

\section{TEST PROCEDURES}

The subjects were instructed to walk on a 25-m smooth, horizontal walkway, with a $2.5-\mathrm{m}$ space at each end of the walkway for acceleration and deceleration. Thus, measurements were performed over a distance of $20 \mathrm{~m}$. Subjects walked the length of this walkway three times at their preferred speeds, wearing shoes that did not influence their gait.

\section{APPARATUS}

We used two kinds of acceleration measurement terminals: One equipped with a smartphone (Xperia S0-01B, Android ${ }^{\mathrm{TM}}$ operating system version $2.1,139 \mathrm{~g}, 119 \times 60 \times 13.1 \mathrm{~mm}$, Sony Ericsson Co., Japan) and the other equipped with a tri-axial accelerometer (model WAA-006, $17 \mathrm{~g}, 38 \times 39 \times 10 \mathrm{~mm}$, ATR-Promotions Co., Japan). The smartphone and the tri-axial accelerometer were taped together. With the method used by Moe-Nilssen and Helbostad, ${ }^{10}$ the terminals were secured over the L3 region, which is close to where the body's center of mass is believed to be located during quiet standing. We developed a gait analysis application and installed it in the smartphone to measure the acceleration of the terminal. This application measured the acceleration of an Android smartphone and immediately displayed the gait analysis results on the smartphone's screen. In our gait analysis, the sampling rate of acceleration measurement for the smartphone was set at SENSOR_DELAY_FASTEST, which is the highest mode listed in the specifications for an Android smartphone. Because the sampling rate was not constant, we adjusted the sampling rate of the acceleration measurement in the smartphone to $0.03 \mathrm{~s}$ during interpolation. In total, 256 samples (7.68 s) of acceleration data were obtained from each measurement terminal and analyzed. For the same reasons as above, the sampling rate of the tri-axial accelerometer was set to $0.03 \mathrm{~s}$.

\section{DATA PROCESSING}

We selected the following gait parameters, according to previous studies: peak frequency $(\mathrm{PF}),{ }^{11}$ root mean square (RMS), ${ }^{10}$ autocorrelation peak (AC), ${ }^{1,12}$ and the coefficient of variance (CV) of the acceleration peak intervals. $^{13,14}$

The PF value indicates the gait cycle, which is the time taken for one step. The RMS value indicates the degree of gait instability; thus, a higher RMS value indicates a lower degree of stability. The AC value indicates the degree of gait balance, so a higher $\mathrm{AC}$ value indicates a greater degree of balance. The CV value indicates the degree of gait variability (i.e., the variability in the elapsed time between the first contacts of two consecutive footfalls). To calculate the gait parameters, we used the absolute values of the tri-axial acceleration data to decrease the influence of the measurement terminal posture. Let $a_{t_{1}: t_{n}}=a_{t_{1}}, a_{t_{2}}, \ldots, a_{t_{n}}$ denote the set of all acceleration absolute values acquired from time $t_{1}$ to $t_{n}$, for $t_{1} \leq t_{n}$. Let $a_{t}$ and $n$, respectively, denote the acceleration absolute value at time $t$ and the number of all acceleration absolute values acquired from time $t_{1}$ to $t_{n}$.

\section{PF DETECTION PROCEDURE}

The PF $f_{p}$ of acceleration data $a_{t_{1}: t_{n}}$ was detected with high accuracy based on the PF candidate $f_{p}^{\prime}$, which was detected from the smoothed acceleration data in order to decrease the influence of the high-frequency measurement noise that accompanied PF detection. The PF detection procedure is shown in Figure 1. First, acceleration data $a_{t_{1}: t_{n}}$ were smoothed using a low-pass filter. Second, the PF candidate, $f_{p}^{\prime}$, was detected where the power spectrum at frequency $f_{p}^{\prime}$ was the highest peak in the frequency space to which the smoothed acceleration data were converted by fast Fourier transformation. Finally, $\operatorname{PF} f_{p}$ was detected in the frequency space to which acceleration data $a_{t_{1}: t_{n}}$ were converted, where the power spectrum of $\operatorname{PF} f_{p}$ had the highest peak around PF candidate $f_{p}^{\prime}$.

\section{PROCEDURE FOR CALCULATION OF RMS}

The RMS of acceleration data $a_{t_{1}: t_{n}}$ was calculated as follows:

$$
R M S=\left(\frac{\int_{t_{1}}^{t_{n}} a(t)^{2} d t}{t_{n}-t_{1}}\right)^{\frac{1}{2}}
$$

Here, let $t_{1}$ and $t_{n}$, respectively, denote the start time and the stop time of our gait analysis measurement.

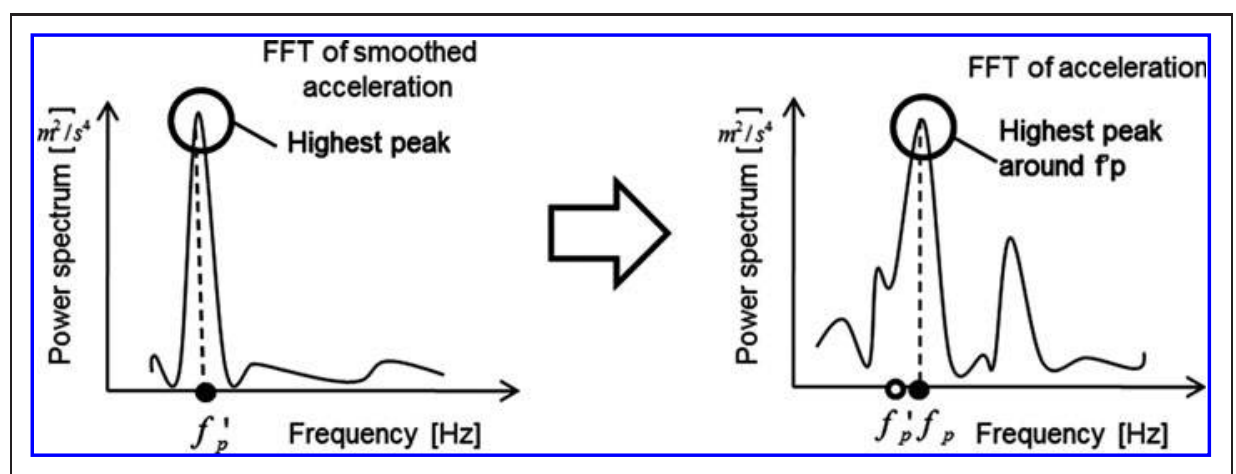

Fig. 1. Peak frequency detection procedure. FFT, fast Fourier transform. 


\section{NISHIGUCHI ET AL.}

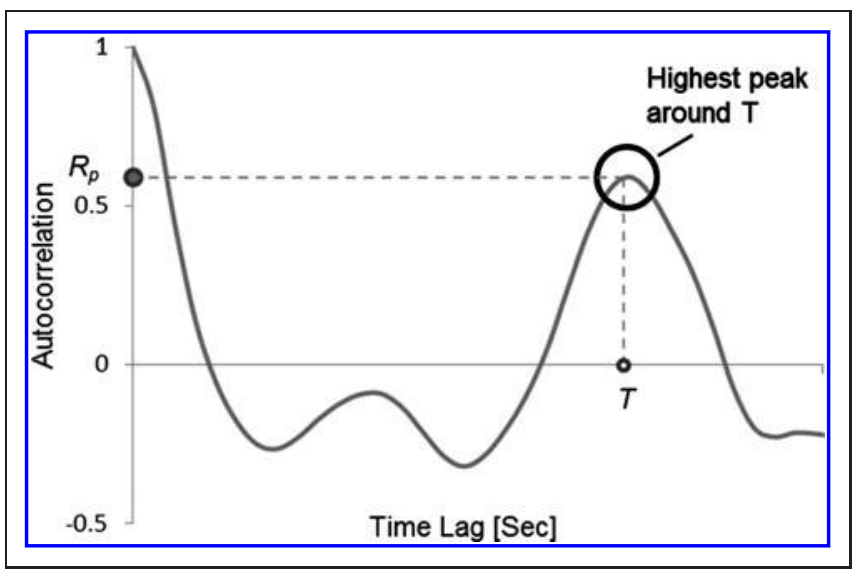

Fig. 2. Autocorrelation peak detection procedure.

\section{PROCEDURE FOR DETECTION OF AC}

The procedure for detection of AC is shown in Figure 2. AC $R_{p}$ from the autocorrelation function was detected by using $\operatorname{PF} f_{p}$. This allowed us to detect AC $R_{p}$ with a high degree of accuracy, based on the hypothesis that the gait cycle is related to the time lag of when AC detection. ${ }^{10}$ The AC detection method was follows. First, the autocorrelation function was calculated from the acceleration data. $a_{t_{1}: t_{n}}$. The autocorrelation function was represented by the sequence of the following autocorrelation coefficients $R_{x x}(k)$ over increasing time lags $k$ :

$$
R_{x x}(k)=\frac{1}{n-k} \sum_{i=1}^{n-k} x_{t_{i}} x_{t_{i+k}}
$$

Here, let $x_{t}$ denote the normalized acceleration data, which was calculated by both the mean $a_{\mathrm{MEAN}}$ and standard deviation $a_{\mathrm{SD}}$ of the acceleration data $a_{t_{1}: t_{n}}$; that is,

$$
x(t)=\frac{a(t)-a_{\mathrm{MEAN}}}{a_{\mathrm{SD}}}
$$

Let $n$ denote the number of acceleration data samples in our gait analysis. Finally, $\mathrm{AC} R_{p}$ was detected as the highest peak around the lag related to gait cycle $T\left(=1 / f_{p}\right)$.

\section{PROCEDURE FOR CALCULATION OF CV}

The CV was calculated by using the group of positive peak time candidates detected in the smoothed acceleration data. This reduced the influence of the high-frequency measurement noise that accompanied positive peak detection. Here, the positive peak indicated the acceleration data with a positive convex shape on the acceleration waveform. First, acceleration data $a_{t_{1}: t_{n}}$ were smoothed using a low-pass filter. Second, each positive peak on the smoothed acceleration waveform was detected as a group of positive peak candidates. These measured times were extracted as a group of positive peak time candidates. Third, each positive peak of acceleration data $a_{t_{1}: t_{n}}$ was detected where each peak was the highest around each positive peak time candidate on the acceleration waveform. The time intervals between the neighboring positive peaks were then calculated. Finally, the $\mathrm{CV}$ was calculated from the mean $t_{\mathrm{MEAN}}$ and standard deviation $t_{\mathrm{SD}}$ of time intervals, as follows:

$$
\frac{t_{\mathrm{SD}}}{t_{\mathrm{MEAN}}}
$$

\section{STATISTICAL ANALYSIS}

The test-retest reliability of the gait analysis performed using the smartphone was assessed using the ICCs $\left(\mathrm{ICC}_{1,1}\right)$ of the values of the gait parameters obtained by the smartphone (PF, RMS, AC, and CV). The criterion-related validity was determined by evaluating the correlation between the gait parameters obtained by the smartphone and the tri-axial accelerometer using Spearman's correlation coefficient $r$. Data were recorded and analyzed using the Statistical Package for the Social Sciences (Windows version 19.0). Statistical significance was considered at $p<0.01$.

\section{Results \\ SUBJECT CHARACTERISTICS}

The subjects were between 18 and 27 years old, with a mean age of $20.9 \pm 2.1$ years. The mean height and weight were $167.3 \pm 7.8 \mathrm{~cm}$ and $60.4 \pm 7.7 \mathrm{~kg}$, respectively. The mean gait speed and cadence were $1.41 \pm 0.03 \mathrm{~m} / \mathrm{s}$ and $121.03 \pm 1.35$ steps/min, respectively.

\section{TEST-RETEST RELIABILITY}

Remarkable consistency was observed in the test-retest reliability of all the gait parameter results obtained by the smartphone $(p<0.001)$ : PF ICC $_{1,1}=0.906,95 \%$ confidence interval (CI) $0.83-$ 0.95; RMS ICC I $_{1}=0.902,95 \%$ CI 0.82-0.95; AC ICC $_{1,1}=0.752,95 \%$ CI 0.55-0.87; and CV ICC I, $_{1}=0.777,95 \%$ CI 0.59-0.89.

\section{CRITERION-RELATED VALIDITY}

The acceleration waveforms of the smartphone and tri-axial accelerometer are shown in Figure 3. All gait parameter results obtained by the smartphone showed considerable and statistically significant correlations with those obtained by the tri-axial accelerometer $(p<0.01)$ (Table 1).

\section{Discussion}

The results of this study indicate that the gait parameters obtained by the smartphone are reliable. Furthermore, the smartphone and the tri-axial accelerometer showed similar acceleration waveforms (Fig. 3). The parameters obtained by the smartphone were considerably correlated with those obtained by the tri-axial accelerometer. Thus, the smartphone with gait analysis application used in this study offers high test-retest reliability and has the capacity to quantify gait parameters as 


\section{GAIT ANALYSIS BY ANDROID-BASED SMARTPHONE}

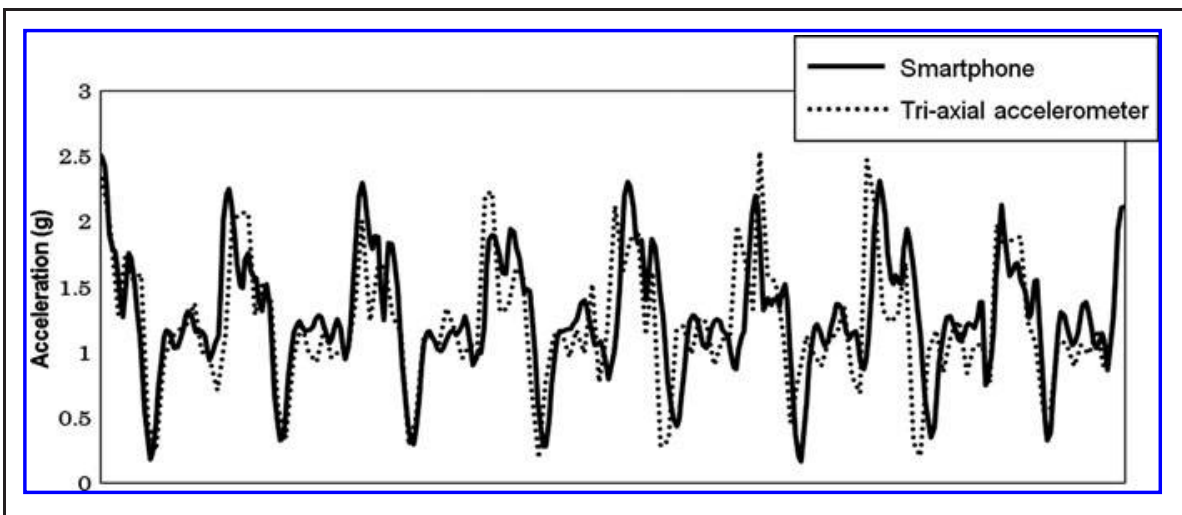

Fig. 3. Acceleration waveforms of the smartphone and tri-axial accelerometer. accelerometer for gait analysis. However, this application appears difficult to manipulate and needs to be improved for greater ease of use. Recently, some researchers have studied the use of smartphones as a self-monitoring or management tool, for example, in cardiac and pulmonary rehabilitation. ${ }^{16,17}$ We consider that such applications can be used for the promotion of public health. In this context, there is a need to develop smartphones equipped with applications such as the one used in this study. Such new medical devices could then be used not only for young people but also for the elderly to prevent falls, to enable self-monitoring activities of orthopedic patients, etc. accurately as a tri-axial accelerometer. The gait parameters used in this study (PF, RMS, AC, and CV) can be used to assess gait patterns from different perspectives. ${ }^{10-14}$ The PF indicates the gait cycle. The RMS indicates the degree of gait instability; the higher the RMS, the lower is the degree of stability. The AC indicates the degree of gait balance; the higher $\mathrm{AC}$, the greater is the degree of balance. The CV indicates the degree of gait variability. It is possible that accidental falls in elderly people could be predicted using these parameters. ${ }^{13,15}$ This indicates that smartphones have the potential to assess the risk of fall and can be used as a new tool for fall prevention in the future.

Smartphones offer several important advantages that are useful for a potential portable medical device. ${ }^{8,9}$ First, smartphones are now ubiquitous devices, and they are less expensive than the conventional accelerometers. Second, they can process and save large amounts of data and convey gait data via both wireless transmission and e-mail. This enables us to assess gait patterns easily in daily life. Third, smartphones are equipped with applications that are flexible and that can be easily improved. For these reasons, a smartphone is more advantageous than a conventional

\begin{tabular}{|c|c|c|c|}
\hline & SMARTPHONE & $\begin{array}{c}\text { TRI-AXIAL } \\
\text { ACCELEROMETER }\end{array}$ & $\begin{array}{c}\text { SPEARMAN'S } \\
\boldsymbol{R}\end{array}$ \\
\hline $\mathrm{PF}(\mathrm{Hz})$ & $2.08(1.82-2.21)$ & $2.08(1.82-2.21)$ & $0.99^{*}$ \\
\hline RMS $(g)$ & $10.86(10.48-12.62)$ & $11.23(10.89-12.37)$ & $0.89^{*}$ \\
\hline$A C$ & $0.81(0.61-0.91)$ & $0.87(0.71-0.95)$ & $0.82^{*}$ \\
\hline $\mathrm{CV}$ & $0.092(0.04-0.18)$ & $0.064(0.02-0.15)$ & $0.85^{*}$ \\
\hline
\end{tabular}

Data are median (range) values.

${ }^{*} p<0.01$ is statistically significant.

$A C$, autocorrelation peak; $C V$, coefficient of variance; $P F$, peak frequency; RMS, root mean square; Spearman's $r$, Spearman's correlation coefficient.

\section{Acknowledgments}

We are grateful to the students of the Department of Human Health Sciences at Kyoto University for their help with data collection.

\section{Disclosure Statement}

Tatsuaki Ito, Shinyo Muto, and Tatsuya Ishihara are employees of NTT Cyber Solutions Laboratories. All the other authors have no conflict of interest.

\section{REFERENCES}

1. Rueterbories J, Spaich EG, Larsen B, et al. Methods for gait event detection and analysis in ambulatory systems. Med Eng Phys 2010;32:545-552.

2. Ornetti P, Maillefert JF, Laroche $D$, et al. Gait analysis as a quantifiable outcome measure in hip or knee osteoarthritis: A systematic review. Joint Bone Spine 2010;77:421-425.

3. Alexander NB, Goldberg A. Gait disorders: Search for multiple causes. Cleve Clin J Med 2005;72:586-600.

4. Hreljac $A$, Marshall RN. Algorithms to determine event timing during normal walking using kinematic data. J Biomech 2000;33:783-786.

5. Menz HB, Latt MD, Tiedemann A, Kwan MMS, Lord SR. Reliability of the GAITRite ${ }^{\circledR}$ walkway system for the quantification of temporospatial parameters of gait in young and older people. Gait Posture 2004;20:20-25.

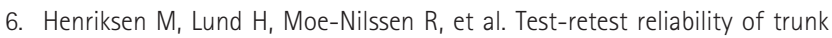
accelerometric gait analysis. Gait Posture 2004;19:288-297.

7. Kavanagh JJ, Menz HB. Accelerometry: A technique for quantifying movement patterns during walking. Gait Posture 2008;28:1-15

8. Rigoberto MM, Toshiyo T, Masaki S. Smart phone as a tool for measuring anticipatory postural adjustments in healthy subjects, a step toward more personalized healthcare. Conf Proc IEEE Eng Med Biol Soc 2010;2010: $82-85$.

9. Lemoyne R, Mastroianni T, Cozza M, et al. Implementation of an iPhone as a wireless accelerometer for quantifying gait characteristics. Conf Proc IEEE Eng Med Biol Soc 2010;2010:3847-3851.

10. Moe-Nilssen R, Helbostad JL. Trunk accelerometry as a measure of balance control during quiet standing. Gait Posture 2002;16:60-68.

11. Auvinet B, Berrut $G$, Touzard $C$, et al. Reference data for normal subjects obtained with an accelerometric device. Gait Posture 2002;16:124-134. 


\section{NISHIGUCHI ET AL.}

12. Moe-Nilssen R, Helbostad JL. Estimation of gait cycle characteristics by trunk accelerometry. J Biomech 2004;37:121-126.

13. Hausdorff JM, Rios DA, Edelberg HK. Gait variability and fall risk in communityliving older adults: A 1-year prospective study. Arch Phys Med Rehabil 2001;82:1050-1056.

14. Beauchet 0 , Allali G, Annweiler $C$, et al. Gait variability among healthy adults: Low and high stride-to-stride variability are both a reflection of gait stability. J Gerontol 2009;55:702-706.

15. Menz HB, Lord SR, Fitzpatrick RC. Acceleration patterns of the head and pelvis when walking are associated with risk of falling in community-dwelling older people. J Gerontol 2003;32:137-142.

16. Worringham C, Rojek A, Stewart I. Development and feasibility of a smartphone, ECG and GPS based system for remotely monitoring exercise in cardiac rehabilitation. PLoS One $2011 ; 6:$ e14669.

17. Marshall A, Medvedev O, Antonov A. Use of a smartphone for improved self-management of pulmonary rehabilitation. Int J Telemed App/ 2008: 753064.
Address correspondence to: Shu Nishiguchi, R.P.T. Department of Physical Therapy Human Health Sciences

Kyoto University Graduate School of Medicine 53 Kawahara-cho, Shogoin, Sakyo-ku

Kyoto 606-8507

Japan

E-mail: shu.n@ks2.ecs.kyoto-u.ac.jp

Received: June 29, 2011

Revised: September 3, 2011

Accepted: September 5, 2011 